\title{
The development and validation of new equations for estimating body fat percentage among Chinese men and women
}

\author{
Xin Liu $^{1}$, Qi Sun ${ }^{2,3}$, Liang Sun ${ }^{1}$, Geng Zong ${ }^{1}$, Ling Lu ${ }^{1}$, Gang Liu ${ }^{1}$, Bernard Rosner ${ }^{2,4}$, \\ Xingwang $\mathrm{Ye}^{1}$, Huaixing $\mathrm{Li}^{1}$ and $\mathrm{Xu} \mathrm{Lin}{ }^{1 *}$ \\ ${ }^{1}$ Key Laboratory of Nutrition and Metabolism, Institute for Nutritional Sciences, Shanghai Institutes for Biological Sciences, \\ Chinese Academy of Sciences and University of Chinese Academy of Sciences, 320 Yueyang Road, Shanghai 200031, \\ People's Republic of China \\ ${ }^{2}$ Channing Division of Network Medicine, Department of Medicine, Brigham and Women's Hospital and \\ Harvard Medical School, Boston, MA, USA \\ ${ }^{3}$ Department of Nutrition, Harvard School of Public Health, Boston, MA, USA \\ ${ }^{4}$ Department of Biostatistics, Harvard School of Public Health, Boston, MA, USA \\ (Submitted 25 February 2014 - Final revision received 20 January 2015 - Accepted 4 February 2015 - First published online 8 April 2015)
}

\begin{abstract}
Equations based on simple anthropometric measurements to predict body fat percentage (BF\%) are lacking in Chinese population with increasing prevalence of obesity and related abnormalities. We aimed to develop and validate BF\% equations in two independent populationbased samples of Chinese men and women. The equations were developed among 960 Chinese Hans living in Shanghai (age 46.2 (SD 5.3) years; $36.7 \%$ male) using a stepwise linear regression and were subsequently validated in 1150 Shanghai residents $(58 \cdot 7$ (SD $6 \cdot 0)$ years; $41.7 \%$ male; $99 \%$ Chinese Hans, $1 \%$ Chinese minorities). The associations of equation-derived BF\% with changes of 6-year cardiometabolic outcomes and incident type 2 diabetes (T2D) were evaluated in a sub-cohort of 780 Chinese, compared with BF\% measured by dual-energy X-ray absorptiometry (DXA; BF\%-DXA). Sex-specific equations were established with age, BMI and waist circumference as independent variables. The BF\% calculated using new sex-specific equations (BF\%-CSS) were in reasonable agreement with BF\%-DXA (mean difference: 0.08 (2 SD 6.64) \%, $P=0.606$ in men; 0.45 ( 2 sD 6.88)\%, $P<0.001$ in women). In multivariate-adjusted models, the BF\%-CSS and BF\%-DXA showed comparable associations with 6-year changes in TAG, HDL-cholesterol, diastolic blood pressure, C-reactive protein and uric acid ( $P$ for comparisons 20.05). Meanwhile, the BF\%-CSS and BF\%-DXA had comparable areas under the receiver operating characteristic curves for associations with incident T2D (men $P=0.327$; women $P=0.159$ ). The $\mathrm{BF} \%$ equations might be used as surrogates for $\mathrm{DXA}$ to estimate $\mathrm{BF} \%$ among adult Chinese. More studies are needed to evaluate the application of our equations in different populations.
\end{abstract}

Key words: Body fat percentage: Equations: Type 2 diabetes: Prospective studies

Obesity, characterised by excessively accumulated body $\mathrm{fat}^{(1)}$, is one of the well-established risk factors for many metabolic diseases, including type 2 diabetes (T2D) ${ }^{(2)}$. BMI has been widely used to define obesity in numerous epidemiological studies because of its simplicity and availability ${ }^{(3)}$. Meanwhile, BMI does not discriminate between fat and lean tissue. Therefore, it may not be a very precise indicator of body fat percentage $(\mathrm{BF} \%)$, which is associated with various metabolically abnormal phenotypes independent of $\mathrm{BMI}^{(4-6)}$. In contrast, among individuals with well-developed musculature, BMI tends to overestimate the BF\% levels ${ }^{(7)}$. Therefore, not only BMI but also $\mathrm{BF} \%$ is important when screening susceptibilities of metabolic outcomes ${ }^{(8)}$. To date, advanced technologies such as isotope dilution, MRI and dual-energy X-ray absorptiometry (DXA) have been employed to objectively measure $\mathrm{BF} \%$; however, their expensive, technologically complex and timeconsuming natures ${ }^{(3)}$ hinder their applications in epidemiological field studies, especially in underdeveloped countries and regions. Skinfold thickness is simpler and cheaper, but is still device-dependent and requires well-trained operators ${ }^{(9)}$.

In recent years, numerous findings have suggested that equations incorporating age, sex, BMI and/or waist circumference

Abbreviations: $\mathrm{BF} \%$, body fat percentage; $\mathrm{BF} \%-\mathrm{C}$, body fat percentage calculated using equations; $\mathrm{BF} \%$-CG, body fat percentage calculated using the general equation; BF\%-CSS, body fat percentage calculated using sex-specific equations; BF\%-DXA, body fat percentage measured by dual-energy X-ray absorptiometry; CRP, C-reactive protein; DXA, dual-energy X-ray absorptiometry; GMO, Gut Microbiota and Obesity; NHAPC, Nutrition and Health of Aging Population in China; SEE, standard error of the estimate; T2D, type 2 diabetes; UA, uric acid; WC, waist circumference. 
(WC) could be alternatively used to predict $\mathrm{BF} \%{ }^{(10-13)}$. Nevertheless, most of those equations were developed in Western populations, and ethnic differences in the relationship between simple anthropometric measurement and actual body adipose content have been suggested by many studies ${ }^{(11,14,15)}$. Compared with Westerners, Asians are known to have higher body fat content at a given BMI or $\mathrm{WC}^{(16)}$, and also tend to accumulate more abdominal fat ${ }^{(17)}$. Even within Asian populations, $\mathrm{BF} \%$ at the same adipose status may vary among Chinese, Thai and Indonesians ${ }^{(15)}$, requiring the development of Chinese specific BF\% equation(s). Given the relationship between adiposity and cardiometabolic outcomes, it is critical to evaluate whether equation-derived $\mathrm{BF} \%$ performs well in the aforementioned associations $^{(13,18)}$. However, as far as we know, the relationship between equation-derived $\mathrm{BF} \%$ and metabolic deteriorations have only been examined in a few cross-sectional studies ${ }^{(13,18)}$, but not in any prospective cohort study.

Therefore, the aims of the present study were (1) to establish equations as alternatives of DXA to evaluate $\mathrm{BF} \%$, based on the anthropometric and demographic data from a population-based Chinese sample; (2) to validate the newly developed equations in another independent Chinese cohort sample for their accuracies and associations with 6-year changes of cardiometabolic risk factors and the risk of developing T2D, in comparison with BF\% measured by DXA (BF\%-DXA).

\section{Methods}

\section{Study participants}

The training sample was from the Gut Microbiota and Obesity (GMO) study, a population-based case-control study conducted among 1059 non-institutionalised Shanghai residents in China. The fieldwork was conducted in 2007-8, and a detailed study design has been described elsewhere ${ }^{(19)}$. Briefly, participants were recruited through advertisements, and the study population consists of both normal-weight $(18 \leq \mathrm{BMI}$ $<24 \mathrm{~kg} / \mathrm{m}^{2}$ ) and overweight/obese (BMI $\geq 24 \mathrm{~kg} / \mathrm{m}^{2}$ ) individuals aged 35-54 years. People were included if they had been living in Shanghai for at least 10 years, but were excluded if they (1) were diagnosed with diabetes, cancer, CHD, stroke, severe kidney or liver diseases, infectious diseases, severe psychological disorders or physical disabilities; (2) used antibiotics for three consecutive days within 3 months before the study; (3) received gastrointestinal surgery within 1 year; or (4) were in pregnancy or lactation period. In the present study, a total of 960 individuals (352 men and 608 women) with complete data of DXA scan were included for the development of the equation.

Validation of our equations was conducted among subjects from the Nutrition and Health of Aging Population in China (NHAPC) study, a population-based prospective study conducted among community-living Chinese aged 50-70 years in Beijing and Shanghai, China. The study design and data collection at baseline and follow-up have been described elsewhere ${ }^{(20,21)}$. In brief, this study was initiated in 2005 , and aimed to investigate the impacts of environmental and genetic factors and their interactions on age-related diseases. The eligible candidates were residents aged 50-70 years and who had been living in Beijing/Shanghai for at least 20 years. Those with any of the following conditions were excluded: (1) severe psychological disorders, physical disabilities, cancer, CVD, Alzheimer's disease, or dementia, within 6 months; or (2) currently diagnosed with communicable diseases. A 6-year follow-up survey was performed in 2011, and all participants were recruited by health workers from local Centers for Disease Control and Prevention and community clinics. Due to the fact that DXA scanner was available only in Shanghai, the DXA data were obtained from 1150 Shanghai participants at baseline, and were used to validate the equations. A total of 233 (20.3\%) participants were lost during the follow-up; eighty-three participants were diagnosed with diabetes at baseline; and another fifty-four participants did not provide fasting blood samples. After excluding these participants, 780 subjects were included in the analyses of prospective associations between $\mathrm{BF} \%$ and cardiometabolic risk factors, and incident T2D. The present study was conducted according to the guidelines laid down in the Declaration of Helsinki, and all procedures involving human subjects/patients were approved by the Institutional Review Board of the Institute for Nutritional Sciences, Chinese Academy of Sciences. Written informed consent was obtained from all the participants.

\section{Data collection}

In both the GMO and the NHAPC studies, a face-to-face interview was conducted, and information of demographic variables (including ethnicity), health status and behaviours (including smoking, alcohol drinking and physical activity) were obtained using standardised questionnaires ${ }^{(19,20)}$. Current alcohol drinking and/or smoking habits were grouped as 'yes' or 'no'. Current smoking referred to daily smoking for $>6$ months and current alcohol drinking referred to people who consumed any alcohol. Physical activity was evaluated by using the International Physical Activity Questionnaire (short last 7-d format) and categorised as low, moderate or high $(<77 \cdot 5,77 \cdot 5-137 \cdot 8$ and $\geq 137.9$ ) based on the metabolic equivalent-hours/week score ${ }^{(22)}$. The anthropometric and body fat measurements of the participants of the two studies were collected through the same standard protocols and devices. With participants wearing light clothes without shoes, body weight was measured to the nearest $0 \cdot 1 \mathrm{~kg}$, and height was measured to the nearest $0 \cdot 1 \mathrm{~cm}$. WC was measured at the mid-point between the lowest rib and the iliac crest to the nearest $0.1 \mathrm{~cm}$, after inhalation and exhalation; while hip circumference was measured at the widest girth of the hip to the nearest $0 \cdot 1 \mathrm{~cm}$, using a plasticcoated fibreglass tape (Grafco 17-1340-2). Blood pressure was determined on the right arm, after a $5 \mathrm{~min}$ rest at minimum, using an electronic blood pressure monitor (Omron HEM-705CP); three measurements were performed, and the mean of the last two was applied in the analyses. Body fat data were measured with whole-body DXA scanner (Hologic QDR-4500), and BF\% was calculated as total body fat mass divided by body weight. BMI was calculated as weight (in $\mathrm{kg}$ ) divided by the squared height (in $\mathrm{m}$ ). For the NHAPC study, overnight fasting blood samples were collected; plasma glucose, TAG, HDL-cholesterol, C-reactive protein (CRP) and 
Table 1. Characteristics of participants in the training and validation samples

(Mean values and standard deviations; number of participants and percentages)

\begin{tabular}{|c|c|c|c|c|c|c|c|c|}
\hline & \multicolumn{4}{|c|}{ GMO study } & \multicolumn{4}{|c|}{ NHAPC study } \\
\hline & \multicolumn{2}{|c|}{ Men ( $n$ 352) } & \multicolumn{2}{|c|}{ Women (n 608) } & \multicolumn{2}{|c|}{ Men ( $n$ 479) } & \multicolumn{2}{|c|}{ Women (n 671) } \\
\hline & $n$ & $\%$ & $n$ & $\%$ & $n$ & $\%$ & $n$ & $\%$ \\
\hline \multicolumn{9}{|l|}{ Age (years) } \\
\hline Mean & $46 \cdot 2$ & & $46 \cdot 2$ & & $59 \cdot 0$ & & $58 \cdot 5$ & \\
\hline SD & $5 \cdot 2$ & & $5 \cdot 3$ & & 5.9 & & $6 \cdot 1$ & \\
\hline \multicolumn{9}{|l|}{ Weight (kg) } \\
\hline Mean & $72 \cdot 6$ & & $62 \cdot 4$ & & $64 \cdot 3$ & & $57 \cdot 3$ & \\
\hline SD & $12 \cdot 3$ & & 11.5 & & $10 \cdot 1$ & & $9 \cdot 6$ & \\
\hline \multicolumn{9}{|l|}{ BMI $\left(\mathrm{kg} / \mathrm{m}^{2}\right)$} \\
\hline Mean & $24 \cdot 7$ & & $24 \cdot 6$ & & 23.5 & & 23.9 & \\
\hline SD & $3 \cdot 7$ & & $4 \cdot 3$ & & $3 \cdot 2$ & & 3.5 & \\
\hline \multicolumn{9}{|l|}{ WC (cm) } \\
\hline Mean & $89 \cdot 4$ & & $82 \cdot 3$ & & $83 \cdot 3$ & & $80 \cdot 0$ & \\
\hline SD & $10 \cdot 8$ & & $10 \cdot 7$ & & $10 \cdot 4$ & & 9.9 & \\
\hline \multicolumn{9}{|l|}{ BF\%-DXA (\%) } \\
\hline Mean & $22 \cdot 8$ & & $32 \cdot 7$ & & $20 \cdot 5$ & & $32 \cdot 5^{\star}$ & \\
\hline SD & $5 \cdot 0$ & & $5 \cdot 0$ & & $5 \cdot 4$ & & $5 \cdot 3$ & \\
\hline Diagnosed with cancer & 0 & - & 0 & - & 1 & 0.2 & 6 & 0.9 \\
\hline Diagnosed with CHD & 0 & - & 0 & - & 16 & 3.4 & 23 & 3.4 \\
\hline Diagnosed with stroke & 0 & - & 0 & - & 11 & $2 \cdot 3$ & 13 & 1.9 \\
\hline Family history of diabetes & 60 & $17 \cdot 1$ & 112 & $18 \cdot 4$ & 48 & $10 \cdot 0$ & 83 & $12 \cdot 4$ \\
\hline Current alcohol drinking & 227 & 64.5 & 121 & $19 \cdot 9$ & 193 & $40 \cdot 3$ & 30 & 4.5 \\
\hline Current smoking & 224 & $63 \cdot 6$ & 11 & 1.8 & 265 & $55 \cdot 3$ & 8 & $1 \cdot 2$ \\
\hline Urban residence & 352 & 100 & 608 & 100 & 210 & 43.8 & 310 & $46 \cdot 2$ \\
\hline \multicolumn{9}{|l|}{ Education level } \\
\hline $0-6$ years & 3 & 0.7 & 8 & $1 \cdot 2$ & 221 & $46 \cdot 1$ & 374 & $55 \cdot 7$ \\
\hline $7-9$ years & 100 & $24 \cdot 3$ & 180 & $27 \cdot 5$ & 139 & $29 \cdot 0$ & 176 & $26 \cdot 2$ \\
\hline$\geq 10$ years & 308 & 74.9 & 466 & $71 \cdot 3$ & 119 & $24 \cdot 8$ & 121 & $18 \cdot 0$ \\
\hline \multicolumn{9}{|l|}{ Physical activity level } \\
\hline Low & 29 & $8 \cdot 2$ & 62 & $10 \cdot 2$ & 30 & $6 \cdot 3$ & 42 & $6 \cdot 3$ \\
\hline Moderate & 178 & $50 \cdot 6$ & 380 & $62 \cdot 5$ & 198 & $41 \cdot 3$ & 322 & $48 \cdot 0$ \\
\hline High & 145 & $41 \cdot 2$ & 166 & $27 \cdot 3$ & 251 & 52.4 & 307 & $45 \cdot 8$ \\
\hline
\end{tabular}

GMO, Gut Microbiota and Obesity; NHAPC, Nutrition and Health of Aging Population in China; WC, waist circumference; BF\%-DXA, body fat percentage measured by dual energy X-ray absorptiometry.

* Comparisons were made for all variables between the two samples, and all of them were significant differences except for women's BF\%-DXA.

uric acid (UA) levels were measured on an automatic analyser (Hitachi 7080) at baseline and at 6-year follow-up.

\section{Definition of type 2 diabetes}

T2D was defined as any of the following conditions: fasting glucose $\geq 7.0 \mathrm{mmol} / \mathrm{l}$; previous diagnosis; use of anti-diabetic medications.

\section{Statistical analysis}

Stepwise linear regression was used to develop sex-specific $\mathrm{BF} \%$ equations after forcing BMI $\left(\mathrm{kg} / \mathrm{m}^{2}\right)$ and WC $(\mathrm{cm})$ into the model. The significance levels of entering the model and being removed were set as $0 \cdot 10$ and $0 \cdot 05$, respectively ${ }^{(23)}$. In addition to $\mathrm{BMI}$ and $\mathrm{WC}$, the following candidate variables were also considered in the sex-specific equations: age; BMI square $\left(\mathrm{kg}^{2} / \mathrm{m}^{4}\right)$; WC square $\left(\mathrm{cm}^{2}\right)$; interaction terms between age and anthropometric variables. The general equation was developed based on the above candidate variables as well as sex and interaction between sex and anthropometric variables. The coefficient of determination $\left(R^{2}\right)$ was used to evaluate the proportion of total variation of BF\%-DXA explained by new equations. Correlations between two variables were computed by the Pearson correlation coefficient. Differences between any two correlations were evaluated by

Table 2. The newly developed equations

\begin{tabular}{|c|c|c|c|}
\hline & Equation & $R^{2 \star}(\%)$ & SEE* $^{*}(\%)$ \\
\hline Men and woment & $\begin{array}{l}\mathrm{BF} \%=-44.65021389+0.43756706 \times \mathrm{BMI}+0.96844999 \times \mathrm{WC}+0.06394571 \times \text { age }+ \\
19.21114033 \times \text { sex }-0.00406036 \times \mathrm{WC}^{2}-0.08813980 \times \text { sex } \times \mathrm{WC}\end{array}$ & $81 \cdot 1$ & 3.02 \\
\hline Men & $\mathrm{BF} \%=-41.92778773+0.33718996 \times \mathrm{BMI}+0.99622038 \times \mathrm{WC}-0.00403169 \times \mathrm{WC}^{2}$ & $66 \cdot 5$ & $2 \cdot 91$ \\
\hline Women & $\begin{array}{l}\mathrm{BF} \%=-22.46354525+0.32551474 \times \mathrm{BMI}+0.87135268 \times \mathrm{WC}+0.00319864 \times \text { age } \times \mathrm{BMl} \\
\quad-0.00408430 \times \mathrm{WC}^{2}\end{array}$ & 61.9 & 3.08 \\
\hline
\end{tabular}

SEE, standard error of the estimate; BF\%, body fat percentage; WC, waist circumference.

* The $R^{2}$ and SEE were generated during the development of the equations, and the $R^{2}$ of general equation was $66.6 \%$ for men and $61.8 \%$ for women.

$\dagger$ Sex $=0$ for men, and sex $=1$ for women 
Table 3. Pearson's correlations between body fat indices and body fat percentage measured by dual-energy X-ray absorptiometry (BF\%-DXA) in the validation sample*

\begin{tabular}{lccc}
\hline & \multicolumn{3}{c}{ BF\%-DXA } \\
\cline { 2 - 4 } & $\begin{array}{c}\text { Total } \\
(n \text { 1150) }\end{array}$ & $\begin{array}{c}\text { Men } \\
(n \text { 479) }\end{array}$ & $\begin{array}{c}\text { Women } \\
(n \text { 671) }\end{array}$ \\
\hline BF\%-CG & 0.905 & 0.790 & 0.761 \\
BF\%-CSS & 0.905 & 0.787 & 0.763 \\
BMI & 0.530 & 0.721 & 0.725 \\
WC & 0.368 & $0.785 \dagger$ & 0.705 \\
Waist:hip ratio & 0.159 & 0.729 & 0.515 \\
Body adiposity index $\ddagger$ & 0.761 & 0.557 & 0.613
\end{tabular}

$\mathrm{BF} \%-\mathrm{CG}$, body fat percentage calculated using the general equation; BF\%-CSS, body fat percentage calculated using sex-specific equations; WC, waist circumference.

* Correlation coefficients were all significant, $P<0.001$.

† Not significantly different from the correlation coefficient between BF\%-DXA and $\mathrm{BF} \%-\mathrm{CSS} / \mathrm{BF} \%-\mathrm{CG}$ in the same column using Wolfe's test.

$\ddagger$ Body adiposity index, hip circumference/height ${ }^{1.5}-18$.

Wolfe's test ${ }^{(24)}$. Bland-Altman analysis ${ }^{(25)}$ was performed to examine the agreement between $\mathrm{BF} \%$ calculated using equations (BF\%-C) and BF\%-DXA. The difference was calculated by subtracting BF\%-DXA from BF\%-C, and the significance levels were tested by paired $t$ test. The mean difference was also considered as an indicator of bias in the comparisons of our equations with others. A generalised linear model was conducted to evaluate the associations between $\mathrm{BF} \%$ and 6-year changes in cardiometabolic risk factors, adjusted for baseline values of age, residence (urban and rural), a certain risk factor (glucose, TAG, HDLcholesterol, blood pressure, CRP or UA), physical activity level (high, moderate or low), education attainment (0-6, 7-9 or $\geq 10$ years), alcohol drinking (yes or no), smoking (yes or no), having cancer, CHD and stroke. To examine whether the associations with cardiometabolic markers were equivalent between $\mathrm{BF} \%$ calculated using sex-specific equations (BF\%-CSS) and BF\%-DXA, the residuals of the indices and changes of cardiometabolic markers were derived by regressing these variables on the aforementioned covariates. The correlations between the residuals of a specific biomarker and the residuals of $\mathrm{BF} \%-\mathrm{CSS}$ and $\mathrm{BF} \%$-DXA were then calculated, respectively. Finally, Wolfe's test was used to compare the two correlation coefficients ${ }^{(24)}$. When analysing the association with longitudinal change of CRP, thirty-eight subjects with CRP levels above $10 \mathrm{mg} / \mathrm{l}$ at baseline or at 6 years of follow-up were excluded. The area under the receiver operating characteristic curve was calculated to evaluate the associations of $\mathrm{BF} \%$-CSS or $\mathrm{BF} \%$-DXA with incident T2D and potential confounders included age, residence, levels of physical activity, education, alcohol drinking, smoking, presence of chronic diseases (cancer, CHD or stroke) and family history of diabetes. The data analysis for the present study was generated using SAS software, version 9.3 of the SAS system for Windows $\times 64$ based systems. Copyright $^{(}$2002-2010 Institute, Inc., SAS and all other SAS Institute, Inc. product or service names are registered as trademarks or trademarks of SAS Institute, Inc. The significance level was set as $P<0.05$ (two-sided).

\section{Results}

\section{Characteristics of subjects}

The characteristics of participants in the GMO study and the NHAPC study (baseline) are presented in Table 1. The BF\%-DXA of the two samples were comparable for women, but not for men (GMO: 22.8 (SD 5.0) \% v. NHAPC: 20.5 (SD 5.4) \%,P<0.05). Both samples represented a wide range of BMI (GMO: $18 \cdot 0-41.6 \mathrm{~kg} / \mathrm{m}^{2}$; NHAPC: $14 \cdot 1-35.6 \mathrm{~kg} / \mathrm{m}^{2}$ ) and BF\%-DXA (GMO: 9.0-47.5\%; NHAPC: $7 \cdot 9-45 \cdot 5 \%$ ).

\section{Developed equations and their accuracies}

Sex-specific and general equations were established in the training sample using stepwise selection in linear regression (Table 2). The $R^{2}$ and standard error of the estimate (SEE) were 66.5 and $2.91 \%$ for men's equation, and 61.9 and $3.08 \%$ for women's equation, respectively. The general equation had an $R^{2}$ of $81.1 \%$ and a SEE of $3.02 \%$.

Validation analyses were performed for both sex-specific equations and the general equation. Herein, BF\%-DXA was strongly correlated with $\mathrm{BF} \%-\mathrm{CSS}$ and $\mathrm{BF} \%$ calculated using the general equation (BF\%-CG; $r 0.905$ for both correlations), while correlations were relatively weaker between BF\%-DXA and anthropometric indices, including BMI, WC, waist:hip ratio and body adiposity index (hip circumference/ height ${ }^{1.5}-18, P$ for Wolfe's test $<0.05$; Table 3 ). When analyses were performed separately by sex, the highest correlation coefficient between $\mathrm{BF} \%-\mathrm{CSS}$ or $\mathrm{BF} \%-\mathrm{CG}$ and $\mathrm{BF} \%-\mathrm{DXA}$ was maintained in women ( $P$ for Wolfe's test $<0.05$ ), but not in men. The scatterplot of BF\%-CSS against BF\%-DXA showed that all dots were near the dashed line (intercept $=0$; slope $=1$ ), suggesting a similarity of the two measures (Fig. 1). Similar dot pattern was also observed in the scatterplot of

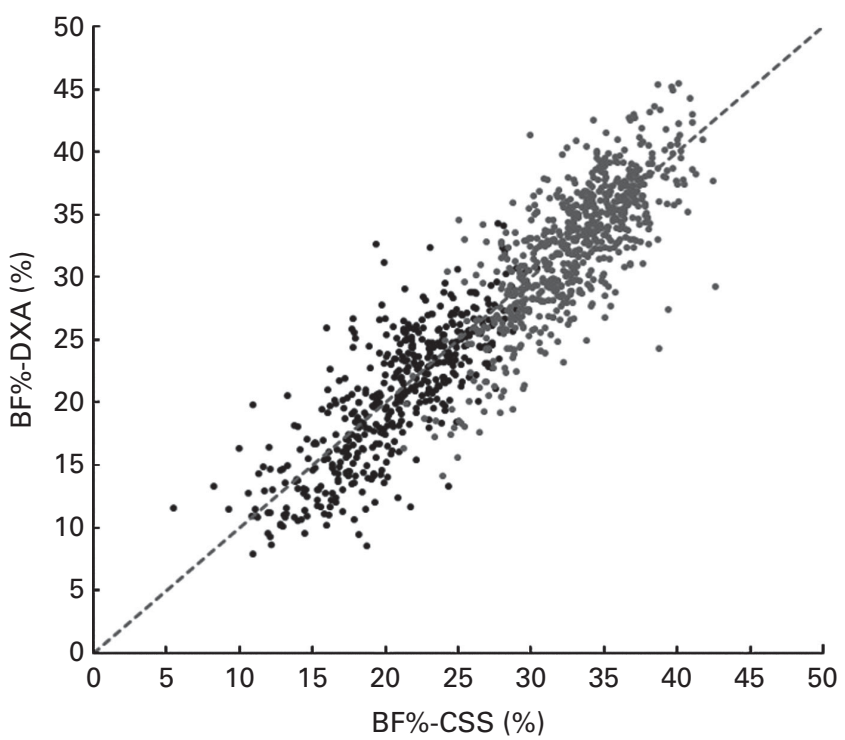

Fig. 1. Agreement between body fat percentage (BF\%) calculated using sex-specific equations (BF\%-CSS) and BF\% measured by dual-energy $X$-ray absorptiometry (BF\%-DXA) in 1150 subjects of the NHAPC study. -.--. Identical values of the two methods; $\bullet$ men; $\bullet$, women. 
Table 4. Bland-Altman analysis of the equations in the validation sample

\begin{tabular}{llcrr}
\hline Equation & Subjects & Mean difference (\%) & $P$ & Limit of agreement (\%)* $^{*}$ \\
\hline General equation & Total, $n$ 1150 & 0.57 & $<0.001$ & $26.23,7.38$ \\
General equation & Men, $n$ 479 & 0.97 & $<0.001$ & $25 \cdot 62,7.55$ \\
General equation & Women, $n$ 671 & 0.29 & 0.029 & $26.62,7.21$ \\
Sex-specific equation & Total, $n$ 1150 & 0.29 & 0.003 & $26.49,7.07$ \\
Sex-specific equation & Men, $n$ 479 & 0.08 & 0.601 & $26.56,6.72$ \\
Sex-specific equation & Women, $n$ 671 & 0.45 & $<0.001$ & $26.42,7.32$ \\
\hline
\end{tabular}

* Limit of agreement $=$ mean difference $-2 \mathrm{SD}$ to mean difference $+2 \mathrm{SD}$.

BF\%-CG against BF\%-DXA (figure not shown). Overall, the result of Bland-Altman analysis indicated that the $\mathrm{BF} \%$-CSS had lower bias than BF\%-CG (Table 4), although they had similar Bland-Altman plots (see online supplementary Fig. S1). The limit of agreement (mean difference) was 0.08 ( $2 \mathrm{SD} 6.64) \%$ for men's equation $(P=0.606)$ and 0.45 (2SD $6.88) \%$ for women's equation $(P<0.001)$. Moreover, the mean difference was smaller in individuals with BMI $\geq 24 \mathrm{~kg} / \mathrm{m}^{2}$ or age $\geq 60$ years than their counterparts (see online supplementary Table S1). In Bland-Altman analysis, our equations also showed lower biases (0.08-0.57\%) than four of the previous equations $(-1 \cdot 24-4.56 \%)$ developed in other populations when age, sex and/or BMI were treated as independent variables (see online supplementary Table S2).

\section{Associations of body fat percentage with changes in} cardiometabolic profiles and incident type 2 diabetes

Among the 780 subjects without baseline diabetes in the NHAPC study, all the cardiometabolic risk factors including fasting plasma glucose, TAG, HDL-cholesterol, CRP and UA increased significantly over the 6-year follow-up, except for diastolic blood pressure in men and systolic blood pressure in women (see online supplementary Table S3). Sex-specific equations were further analysed for the associations with metabolic markers, given their better performance in BlandAltman analysis. In the multivariate-adjusted model (adjusted for age, residence, physical activity level, education attainment, alcohol drinking, smoking, and having cancer, CHD or stroke), the $\mathrm{BF} \%$-CSS and $\mathrm{BF} \%$-DXA showed a comparable relationship with baseline levels of TAG, CRP and UA in both men and women (see online supplementary Table S4). Compared with $\mathrm{BF} \%$-DXA, the $\mathrm{BF} \%$-CSS was more strongly associated with glucose in men $(P=0.002)$, and was more strongly associated with HDL-cholesterol $(P=0.033)$, systolic blood pressure $(P=0.009)$ and diastolic blood pressure $(P=0.012)$ in women. Furthermore, BF\%-CSS and BF\%-DXA were largely comparable regarding the associations with 6-year change of those markers, except that the association between the BF\%-CSS and 6-year change of systolic blood pressure was stronger in women ( $P=0.028$; Table 5). Among the 780 participants, $18.0 \%$ of them developed T2D over the 6-year period. Area under the receiver operating characteristic curves were calculated to evaluate the associations of BF\%-CSS or BF\%-DXA with incident T2D. The area under the receiver operating characteristic curves were $0.62(95 \%$ CI $0.53,0.71$ ) for BF\%-CSS and 0.59 (95\% CI $0.50,0.68$ ) for
BF\%-DXA in men $(P$ for comparison $=0.327)$, and $0.66(95 \%$ CI $0.59,0.72)$ for $\mathrm{BF} \%$-CSS and 0.64 (95\% CI $0.58,0.70)$ for BF\%-DXA in women $(P$ for comparison $=0.159)$ (see online supplementary Fig. S2).

\section{Discussion}

In the present study, we developed new equations to estimate BF\% among 960 Chinese with a broad range of adiposity levels, and also conducted validation analyses in another independent Chinese cohort population. The validation analyses showed that BF\%-CSS was in reasonable agreement with BF\%-DXA. Meanwhile, BF\%-CSS and BF\%-DXA had comparable associations with changes in a series of cardiometabolic risk factors and incident T2D over 6 years. To our knowledge, this is thus by far the largest investigation that has developed $\mathrm{BF} \%$ equations in Asian populations, and the findings from the present study also provided new information on the associations between equation-based $\mathrm{BF} \%$ with changes in cardiometabolic risk factors and incident T2D over several years.

To clearly understand more detailed relationships between obese phenotypes and health status, not only BMI but also $\mathrm{BF} \%$ needs to be evaluated in large-scale population studies. However, it is not cost-effective and time-efficient to apply standard devices such as MRI and DXA for BF\% measurement for large epidemiological surveys. In such circumstance, equations based on anthropometry could be the simplest surrogate; however, it was proved to be highly ethnic-specific due to considerable variations in body composition and relationships between $\mathrm{BF} \%$ and $\mathrm{BMI}$ or WC between different ethnic groups $^{(11,14,15)}$. As the largest developing country, China has experienced rapid urbanisation and changes in dietary and lifestyle choices in the past decades ${ }^{(26)}$. Accompanied with the changes, it is estimated that one-fifth of the world's overweight or obese people were Chinese ${ }^{(27)}$. Unfortunately, few studies have evaluated $\mathrm{BF} \%$ and related health consequences using equation(s) generated from Chinese population so far. One skinfold-based $\mathrm{BF} \%$ equation was reported by Kwok et al. ${ }^{(28)}$ based on the data of 613 Hong Kong residents aged 69-82 years. Apparently, skinfold measurement is more accessible than DXA or MRI method; however, its device-dependency and requiring skilful professionals are also not practical in dealing with large sample sizes. Moreover, the narrow age range in the subjects could limit the application of their equation to other Chinese populations. In contrast, the current $\mathrm{BF} \%$ equation only included age, sex, BMI and WC as independent variables, all of which are routinely collected in epidemiological 
studies. Thus, our new, validated equations may facilitate the estimation of $\mathrm{BF} \%$ in large-scale epidemiological studies, specifically among Chinese people.

Our validation analyses in 1150 subjects of the NHAPC study showed that BF\%-CSS was highly correlated with BF\%-DXA. Meanwhile, the bias in women's equation was smaller than some of the previous validation analyses of BMI or WC-derived equations from studies in Americans (White and Black), Spanish, Netherlander, Swedish and Japanese (mean differences $-1 \cdot 0-2 \cdot 9 \%)^{(11,13,29-31)}$. Moreover, there was no significant bias in the equation for men. Those results suggested a satisfactory accuracy of our equations for the purposes of epidemiological assessment. In contrast, the $R^{2}$ of our sex-specific equations (66.5\% for men and $61.9 \%$ for women) were lower than that of our general equation $(81 \cdot 1 \%)$. This discrepancy may be partially interpreted by the fact that sex per se accounted for considerable $\mathrm{BF} \%$ variances in the whole population, due to sex hormonal impact on body fat storage ${ }^{(32,33)}$. The combined data set $(7 \cdot 9-45 \cdot 5 \%)$ and separated data sets $(7 \cdot 9-34.3 \%$ in men; $14 \cdot 2-45.5 \%$ in women) had different ranges of dependent variable (BF\%), which might also affect the magnitude of $R^{2}$ in linear regression ${ }^{(34)}$. Therefore, a low $R^{2}$ may not necessarily indicate poor performance. The SEE could also provide information of estimated accuracy ${ }^{(35)}$. In fact, our sex-specific equations have similar SEE (approximately 3\%) compared with our general equation, as well as the equations developed by others ${ }^{(13,15,30)}$.

Previously, several studies have focused on the relationships between estimated $\mathrm{BF} \%$ and cardiometabolic risk factors, and suggested the usefulness of $\mathrm{BF} \%$ equation. For instance, Gomez-Ambrosi et al. ${ }^{(13)}$ reported that their equation-derived $\mathrm{BF} \%$ exhibited better correlations with blood lipid profile, blood pressure or insulin resistance indices than using BMI or WC alone in 634 diabetic patients. However, that study did not compare estimated $\mathrm{BF} \%$ with objectively measured $\mathrm{BF} \%$ with regard to their associations with obesity-related risk factors; thus, it remains unclear whether the two approaches were interchangeable on those associations. In another crosssectional study of 130 adolescents, Steinberger et al. ${ }^{(18)}$ found that BF\% either estimated by Slaughter's equation or measured by DXA showed comparable correlations with cardiovascular risk factors including glucose utilisation, systolic blood pressure, HDL-cholesterol and insulin. However, no data were available regarding longitudinal associations between estimated $\mathrm{BF} \%$ and changes of risk factors so far. In the present study, we further provided a novel evidence that baseline BF\%-CSS and BF\%-DXA have similar strength in terms of their associations with 6-year changes of diastolic blood pressure, TAG, HDL-cholesterol, glucose, inflammatory markers and UA, as well as with 6-year incident T2D.

Strengths of the present study included the following: (1) the new equations were developed from a large population-based sample with broad ranges of $\mathrm{BMI}$ and $\mathrm{BF} \%$, and the validation was conducted in an independent and large-scale cohort sample; (2) based on a prospective study design, we simultaneously documented associations with longitudinal changes of metabolic risk factors for both BF\%-C and BF\%-DXA for the 
first time. However, all the participants in our training sample and $99 \%$ of participants in our validation sample are Chinese Hans. It remains to be confirmed whether our equations are applicable for other Chinese minority groups. Meanwhile, our validation sample only included Shanghai residents aged 50-70 years, and it is also to be confirmed in more future studies whether or to what extent our new equations could be generalised to nationwide Chinese or other Asian populations. Although DXA was frequently used as a reference method in $\mathrm{BF} \%$ estimation ${ }^{(36)}$, its accuracy could be affected by the content of total body water ${ }^{(37)}$. Since no data of total body water and body density were available, it was not possible to apply four-compartment model in present study.

In conclusion, anthropometry-based $\mathrm{BF} \%$ equations were developed and validated for Chinese men and women. The new equations might serve as a useful tool in large-scale epidemiological surveys to estimate $\mathrm{BF} \%$ among Chinese adults. More studies are needed to evaluate the application of our equations in different populations.

\section{Supplementary material}

To view supplementary material for this article, please visit http://dx.doi.org/10.1017/S0007114515000616

\section{Acknowledgements}

The authors thank Dr Jing Wang, Dr Hongyu Wu, Dr Wei Gan, Jingwen Zhu, He Zheng, Qianlu Jin and Yiqing Wang from the Institutes for Biological Sciences, Chinese Academy of Sciences and University of Chinese Academy of Sciences for their help in various stages of the present study.

The present study was supported by The Ministry of Science and Technology of China (2012CB524900), National Natural Science Foundation of China (81321062), the Knowledge Innovation Program of Shanghai Institutes for Biological Sciences, Chinese Academy of Sciences (2013KIP107) and the SA-SIBS Scholarship Program. These funding sources had no role in the study design, data collection, data analysis and the interpretation of the results.

The authors' contributions are as follows: X. Liu substantially contributed to the acquisition and analyses of the data, and drafted the manuscript; L. S., G. L., L. L., G. Z., X. Y. and H. L. contributed to data collection; Q. S. and B. R. substantially contributed to the conception of the study design, data analysis and revising of the manuscript; $X$. Lin made significant contributions to the study design, supervised data collection and interpretation, and revised the manuscript. All the authors involved in the writing of this article and approved the final content of the manuscript.

None of the authors has any conflict of interest to declare.

\section{References}

1. Deurenberg P, Deurenberg-Yap M \& Guricci S (2002) Asians are different from Caucasians and from each other in their body mass index/body fat per cent relationship. Obes Rev 3, 141-146.
2. Pi-Sunyer FX (2002) The obesity epidemic: pathophysiology and consequences of obesity. Obes Res 10, Suppl. 2, 97S-104S.

3. Wells JC \& Fewtrell MS (2006) Measuring body composition. Arch Dis Child 91, 612-617.

4. Shea JL, King MT, Yi Y, et al. (2012) Body fat percentage is associated with cardiometabolic dysregulation in BMIdefined normal weight subjects. Nutr Metab Cardiovasc Dis 22, 741-747.

5. Kim JY, Han SH \& Yang BM (2013) Implication of high-bodyfat percentage on cardiometabolic risk in middle-aged, healthy, normal-weight adults. Obesity (Silver Spring) 21, 1571-1577.

6. Oliveros E, Somers VK, Sochor O, et al. (2014) The concept of normal weight obesity. Prog Cardiovasc Dis 56, 426-433.

7. Prentice AM \& Jebb SA (2001) Beyond body mass index. Obes Rev 2, 141-147.

8. Gomez-Ambrosi J, Silva C, Galofre JC, et al. (2011) Body adiposity and type 2 diabetes: increased risk with a high body fat percentage even having a normal BMI. Obesity (Silver Spring) 19, 1439-1444.

9. Ellis KJ (2001) Selected body composition methods can be used in field studies. J Nutr 131, 1589S-1595S.

10. Lean ME, Han TS \& Deurenberg P (1996) Predicting body composition by densitometry from simple anthropometric measurements. Am J Clin Nutr 63, 4-14.

11. Jackson AS, Stanforth PR, Gagnon J, et al. (2002) The effect of sex, age and race on estimating percentage body fat from body mass index: The Heritage Family Study. Int J Obes Relat Metab Disord 26, 789-796.

12. Martarelli D, Martarelli B \& Pompei P (2008) Body composition obtained from the body mass index: an Italian study. Eur J Nutr 47, 409-416.

13. Gomez-Ambrosi J, Silva C, Catalan V, et al. (2012) Clinical usefulness of a new equation for estimating body fat. Diabetes Care 35, 383-388.

14. Wang J, Thornton JC, Russell M, et al. (1994) Asians have lower body mass index (BMI) but higher percent body fat than do whites: comparisons of anthropometric measurements. Am J Clin Nutr 60, 23-28.

15. Deurenberg P, Yap M \& van Staveren WA (1998) Body mass index and percent body fat: a meta analysis among different ethnic groups. Int J Obes Relat Metab Disord 22, 1164-1171.

16. Lear SA, Humphries KH, Kohli S, et al. (2007) The use of BMI and waist circumference as surrogates of body fat differs by ethnicity. Obesity (Silver Spring) 15, 2817-2824.

17. Lear SA, Humphries KH, Kohli S, et al. (2007) Visceral adipose tissue accumulation differs according to ethnic background: results of the Multicultural Community Health Assessment Trial (M-CHAT). Am J Clin Nutr 86, 353-359.

18. Steinberger J, Jacobs DR, Raatz S, et al. (2005) Comparison of body fatness measurements by BMI and skinfolds $v s$ dual energy X-ray absorptiometry and their relation to cardiovascular risk factors in adolescents. Int J Obes (Lond) 29, $1346-1352$.

19. Sun L, Yu Z, Ye X, et al. (2010) A marker of endotoxemia is associated with obesity and related metabolic disorders in apparently healthy Chinese. Diabetes Care 33, 1925-1932.

20. Ye X, Yu Z, Li H, et al. (2007) Distributions of C-reactive protein and its association with metabolic syndrome in middle-aged and older Chinese people. J Am Coll Cardiol 49, 1798-1805.

21. Zong G, Zhu J, Sun L, et al. (2013) Associations of erythrocyte fatty acids in the de novo lipogenesis pathway with risk of metabolic syndrome in a cohort study of middle-aged and older Chinese. Am J Clin Nutr 98, 319-326. 
22. Group TIPAQ (2005) Guidelines for data processing and analysis of the International Physical Activity Questionnaire (IPAQ). http://www.ipaq.ki.se/scoring.pdf (accessed May 2005).

23. Kuk JL, Katzmarzyk PT, Nichaman MZ, et al. (2006) Visceral fat is an independent predictor of all-cause mortality in men. Obesity 14, 336-341.

24. Rosner BA (2006) Fundamentals of Biostatistics, 6th ed Belmont, CA: Thomson-Brooks/Cole.

25. Bland JM \& Altman DG (1986) Statistical methods for assessing agreement between two methods of clinical measurement. Lancet 1, 307-310.

26. Hu FB, Liu Y \& Willett WC (2011) Preventing chronic diseases by promoting healthy diet and lifestyle: public policy implications for China. Obes Rev 12, 552-559.

27. Wu Y (2006) Overweight and obesity in China. BMJ 333 $362-363$

28. Kwok T, Woo J \& Lau E (2001) Prediction of body fat by anthropometry in older Chinese people. Obes Res $\mathbf{9}$, 97-101.

29. Minematsu K, Takamura N, Goto K, et al. (2011) A proposed method for the evaluation of body fat in Japanese adults that predicts obesity. Nutr Res 31, 113-121.
30. Deurenberg P, Weststrate JA \& Seidell JC (1991) Body mass index as a measure of body fatness: age- and sex-specific prediction formulas. BrJ Nutr 65, 105-114.

31. Larsson I, Henning B, Lindroos AK, et al. (2006) Optimized predictions of absolute and relative amounts of body fat from weight, height, other anthropometric predictors, and age 1. Am J Clin Nutr 83, 252-259.

32. Geer EB \& Shen W (2009) Gender differences in insulin resistance, body composition, and energy balance. Gend Med 6, Suppl. 1, 60-75.

33. Barreira TV, Harrington DM, Staiano AE, et al. (2011) Body adiposity index, body mass index, and body fat in white and black adults. JAMA 306, 828-830.

34. Barrett JP (1974) Coefficient of determination - some limitations. Am Stat 28, 19-20.

35. Hennig C \& Cooper D (2011) Brief communication: the relation between standard error of the estimate and sample size of histomorphometric aging methods. Am J Phys Anthropol 145, 658-664.

36. Plank LD (2005) Dual-energy X-ray absorptiometry and body composition. Curr Opin Clin Nutr Metab Care 8, 305-309.

37. Laskey MA (1996) Dual-energy X-ray absorptiometry and body composition. Nutrition 12, 45-51. 